(RESEARCH ARTICLE)

\title{
Selection of optimal fluid for refrigeration cycles
}

\author{
Ayman G. A. Essa * and Adil A. Mohamed \\ ${ }^{1}$ Department of chemical engineering, Karary University, Sudan.
}

Publication history: Received on 01 December 2020; revised on 09 December 2020; accepted on 11 December 2020

Article DOI: https://doi.org/10.30574/wjaets.2020.1.2.0031

\begin{abstract}
Refrigeration and air-conditioning play an important role in our life and industrial applications. They have great impact on our life. They have also contributed to the world's major environmental issues like ozone layer depletion and global warming. Common refrigerants such as CFCs and HCFCs which are working as fluid in refrigeration cycles have unfavorable environmental impacts and this has brought about concerns and regulations prohibiting their production and use as refrigerants by the year 2030. The development of different refrigerants over time took place based on safety and environmental impact issues. This paper, presents the selection of optimal working fluids for Vapor-Compression Refrigeration Cycle (VCRs) based on computer aided molecular design (CAMD) and process optimization techniques. The resulting methodology utilizes from CAMD for the generation of optimum working fluid candidates. Candidates were evaluated as alternative refrigerants for the R134a refrigerating system through simulation using Aspen Hysys V8.0, with restricted by priority coefficient of performance COP, environmental and safety criteria. Ethyl trifluoromethyl ether 1,1,1-TrifluoroButane (new refrigerant) shows a good environmental and toxicity data also have high COP, 4.5, 2.7 respectively and were favored amongst the studied refrigerants as the choice alternative refrigerants to replace R134a. The methodology systematically identified conventional molecular structures that enable optimum VCRs process performance.
\end{abstract}

Keywords: Ozone depletion Potential, Global warming Potential, Vapor-Compression Refrigeration Cycle (VCRs), Computer Aided Molecular Design (CAMD).

\section{Introduction}

A refrigerant is a substance or mixture, usually a fluid, used in a heat pump and refrigeration cycle. In most cycles it undergoes phase transitions from a liquid to a gas and back again. Hydrochlorofluorocarbons, chlorofluorocarbons and Fluorocarbons, especially chlorofluorocarbons. Other common refrigerants used in various applications are ammonia, sulfur dioxide, and non-halogenated hydrocarbons such as propane. Early mechanical refrigeration systems employed sulfur dioxide, methyl chloride and ammonia. Being toxic, sulfur dioxide and methyl chloride rapidly disappeared from the market with the introduction of CFCs. Occasionally; one may encounter older machines with chloromethane or dichloromethane (called carrene in the trade).

Scientists observed as early as 1974 that the rampant use of chlorofluorocarbons (CFCs) is destructive to stratospheric ozone. The depletion of the ozone layer, which shields the earth from the harmful effects of the sun's ultraviolet radiation, is caused by chlorine molecules in so-called ozone-depleting substances that are migrated to the stratosphere and then react catalytically with ozone, thus destroying it. Besides participating in the destruction of stratospheric ozone, the release of CFCs may also contribute to global warming, which means that CFCs influence the reflection of infrared radiation from the surface of the earth thus causing global climate change. Thus, the production of CFCs and other halogenated organic compounds such as 1,1,1 trichloroethane and carbon tetrachloride are regulated to phase out at the end of 1995 under the terms of the Montreal Protocol [1,2].

\footnotetext{
${ }^{*}$ Corresponding author: Ayman. G. Ahmed

Department of chemical engineering, Karary University, Sudan.
}

Copyright (c) 2020 Author(s) retain the copyright of this article. This article is published under the terms of the Creative Commons Attribution Liscense 4.0. 
Due to the extensive applications of CFCs in industrial processes including flexible/rigid foam manufacture and solvent cleaning operations, replacements for CFCs and some halogenated organic compounds need to be developed based on the economic and environmental benefits. Hydrochlorofluorocarbons (HCFCs), because of their similar physiochemical properties to those of CFCs, have been used as interim replacements for CFCs. Although HCFCs contain chlorine, the release of this chlorine to the stratosphere is expected to be small, because HCFCs are readily reacted by hydroxyl radicals in the troposphere. Further, the presence of hydrogen in HCFCs causes them to have shorter atmospheric lifetimes and thus may also contribute to global warming. According to the Montreal Protocol, a virtual phase-out of HCFCs is scheduled by $2020[3,4]$.

Another new class of fluorocarbon refrigerants called hydrofluoro-olefin (HFO) with potential for reduced GWP have been developed. Their primary advantage, other than their low GWP, is that they can be used with existing refrigeration system designs. This is good for the industry and their customers, but it is still a fluorinated gas. There is growing political pressure to regulate it out of production and force the industry to develop an even lower-impact refrigeration technology.

The uses of natural fluids have appealed transformed interest during the last decade. Among the natural refrigerants, hydrocarbons have the potentiality to be the replacement of the existing refrigerants. Studies revealed that some of the HCs showed promising and interesting thermal and physical properties. HCs have the high latent heat of vaporization. So these are considered as an alternative to the existing refrigerants [5]. Vapor compression systems are widely used in many cases. It requires huge amount of energy and creates the less sustainable environment. Most of the energy is utilized for lighting, heating, cooling and air conditioning in our daily lifestyle. Which is one of the key source of energy utilization [6-7]. Many of the researchers are believing that HCs can be easily adopted as an alternative refrigerant. They found to have the obvious advantages of being recognized as environmentally conscious and outstanding energy savings are also obtained. The only disadvantage of HCs is, its flammability that relative to other refrigerants. The flammability issue can be minimized by mixing HCs with a small amount of HFCs together for the commercial refrigeration where a large number of charges are essential [5].

The main objective is to selection of optimal fluids flowing in Vapor-Compression Refrigeration Cycle alternative to R134a refrigerant. These fluids will be considered as optimal coefficient of performance, ozone depletion potential and global warming potential. While working fluids were essentially chosen among pure species until recently, the use of multicomponent mixtures (and in particular, of binary mixtures) including low-GWP "green" substances and low ODP is seen as a promising way. To achieve the above aim the following steps will be followed:

Use CAMD software to obtain chemical molecular with desirable physical, chemical, thermodynamic, safety properties that are already known.

Applied molecular structure which obtained from CAMD in Simulate Ideal Vapor-Compression Refrigeration Cycle in HYSYS.

Calculate the COP and parameter of cycle and make comparison between etch molecular.

Chose the molecular which has the best result and specify as alternative to R134a.

\section{Material and methods}

\subsection{Introduction}

Determines the structure and constitution of a system of single or species that satisfies a set of desired properties and functions. The product specifications are defined based on the product needs and may include several types of specifications, such as physicochemical, thermophysical properties, environmental and safety properties.

\subsection{Molecular Properties}

There are numerous properties that should be considered for the design and selection of working fluids for VaporCompression Refrigeration Cycle processes [8,9]. Several of them are considered in this work to illustrate the implementation of the proposed methodology, which is independent of the selected properties as it can handle any desired performance measure or constraint. Such properties are summarized in Table 1 and elaborated as follows: 
The density $(\rho)$ of the working fluid must be high either in the liquid or vapor phase. High liquid or vapor density results to increased mass flowrate and equipment of reduced size [10]. In this work we consider only liquid density.

The latent heat of vaporization ( $\mathrm{Hu}$ ) of the working fluid must be high for numerous reasons. A study presented in [9] notes that high $\mathrm{Hu}$ enables most of the available heat to be added during the phase change operation, hence avoiding the need to regulate the superheating and expansion of the vapor through regenerative feed heating in order to enable higher efficiency. Furthermore, high latent heat is associated with a near vertical saturated vapor line of the fluid, which results to reduced moisture during the expansion as well as avoids the necessity to condense a superheated fluid. The vertical saturation vapor line indicates a fluid that expands between the wet vapor region and the superheated region of a temperature-entropy diagram [9]. Few researchers [10] suggest that Hu must be low to void the selection of fluids that form moisture when saturated vapor is expanded, namely "wet" fluids. This work targets the design of molecules with high $\mathrm{Hu}$ at the CAMD stage and implements an upper limit at the liquid fraction observed at the compressor outlet during vapor compression refrigeration system operation, to avoid the selection of "wet" working fluids.

The liquid heat capacity $\left(\mathrm{C}_{\mathrm{L}}\right)$ of the working fluid must be low because it enables a near vertical saturated liquid line, which has a similar effect to the vapor compression refrigeration system process [11].

The viscosity $(\mu)$ of the working fluid should be maintained low in both liquid and vapor phases in order to achieve a high heat transfer coefficient with reduced power consumption [12].

The thermal conductivity $(\lambda)$ must be high in order to achieve high heat transfer coefficients in both the employed condensers and vaporizers [12].

The melting point temperature $\left(\mathrm{T}_{\mathrm{m}}\right)$ should be lower than the lowest ambient operating temperature in order to ensure that the working fluid will remain in the liquid phase.

The critical temperature $\left(\mathrm{T}_{\mathrm{c}}\right)$ should be higher than the maximum cycle operating temperature, as we only consider subcritical operations in this work.

The ozone depletion potential (ODP) is an index that determines the relative ability of chemical substances to destroy ozone molecules in the stratosphere, hence working fluids with low or zero ODP are required. Data available in published literature are used to determine ODP.

The global warming potential (GWP) is an index that determines the potential contribution of a chemical substance to global warming. The calculation of GWP depends on numerous complex factors and is mostly possible either through experimental data for a particular substance or through generic guidelines, data available in published literature are used to determine GWP.

The determination of the toxicity (C) of the designed working fluids is important for human safety reasons. Data available in published literature are used to determine toxicity [13].

The flammability (F) is an index used to assess the flammability characteristics of the designed working fluids. Data available in published literature are used to determine flammability [14].

The maximum operating pressure $\left(\mathrm{P}_{\max }\right)$ required in the VCRs should generally be low because high pressure processes require the use of expensive equipment.

The mass flowrate $\left(\mathrm{m}_{\mathrm{f}}\right)$ of the working fluid should also be low in order to maintain reduced operating costs.

The critical pressure $\left(\mathrm{P}_{c}\right)$ of the working fluid should be higher that $\mathrm{P}_{\max }$, as only sub-critical operations are considered in this work. 
Table 1 Properties considered as performance measures for the design of working fluids.

\begin{tabular}{|c|c|c|c|}
\hline Thermodynamic & Environmental & Safety & Process-related \\
\hline 1 Density $(\rho)$ & 8 Ozone depletion & 10 Toxicity (C) & 12 Maximum operating \\
\hline 2 Latent heat of vaporization (Hu) & & 11 Flammability (F) & pressure $(P \max )$ \\
\hline 3 Liquid heat capacity $\left(\mathrm{Cp}_{\mathrm{L}}\right)$ & 9 Global warming & & 13 Mass flowrate $\left(\mathrm{m}_{\mathrm{f}}\right)$ \\
\hline 4 Viscosity $(\mu)$ & & & 14 Critical pressure $\left(\mathrm{P}_{c}\right)$ \\
\hline 5 Thermal conductivity $(\lambda)$ & & & \\
\hline 6 Melting point temperature $\left(\mathrm{T}_{\mathrm{m}}\right)$ & & & \\
\hline 7 Critical temperature $\left(T_{c}\right)$ & & & \\
\hline
\end{tabular}

\subsection{Computer Aided Product Design Framework}

The objective is to find a refrigerant as good as or better than R134a. This is defined as a refrigerant with a larger heat of vaporization and with a smaller liquid heat capacity than that of R134a. The replacement refrigerant obtained should be analyzed with respect to environmental properties. One important consideration in material replacement is the desire to use existing equipment and processing technology. This requires that both the old and the substitute material should have similar transport and other properties such as heat capacity and vapor pressure.

In this section, the computer-aided framework for chemical product design is presented. The framework is composed of four sequential steps that enable the formulation and solution of various types of CAMD problems based on selection of appropriate solution strategies. The four steps with their associated methods, tools, and information flow are shown in Fig. 1. Each step is briefly described, together with its required databases, model libraries, and tools in the following text.

\subsubsection{Step 1 Problem Definition.}

In this step, the chemical product needs are defined. These are translated into target properties and target specifications and the target property values are set. The problem definition includes the needs, chemical product type, and the process and/or application of the chemical product. It is important to specify this as clearly and concisely as possible. The needs include target properties and environmental impact of the chemical product. The chemical product type includes a combination of the four types of properties, depending on the molecular design problem. A database is used to help identifying the needs for specific products. The needs are translated into target properties. Target property values are set in terms of lower and upper bounds or as fixed values. These are derived from the needs and specifications on the product.

The refrigerant to be designed is sought to have the same or better performing target properties. In addition, it should be able to function under similar operating conditions as typical systems that use R134a. Therefore, the new refrigerant should have similar or better environmental properties and similar or improved thermodynamic properties. Analysis of thermodynamic properties requires the consideration of both the molecular thermodynamic properties and the performance of the refrigerant in the application process (the refrigeration cycle). 


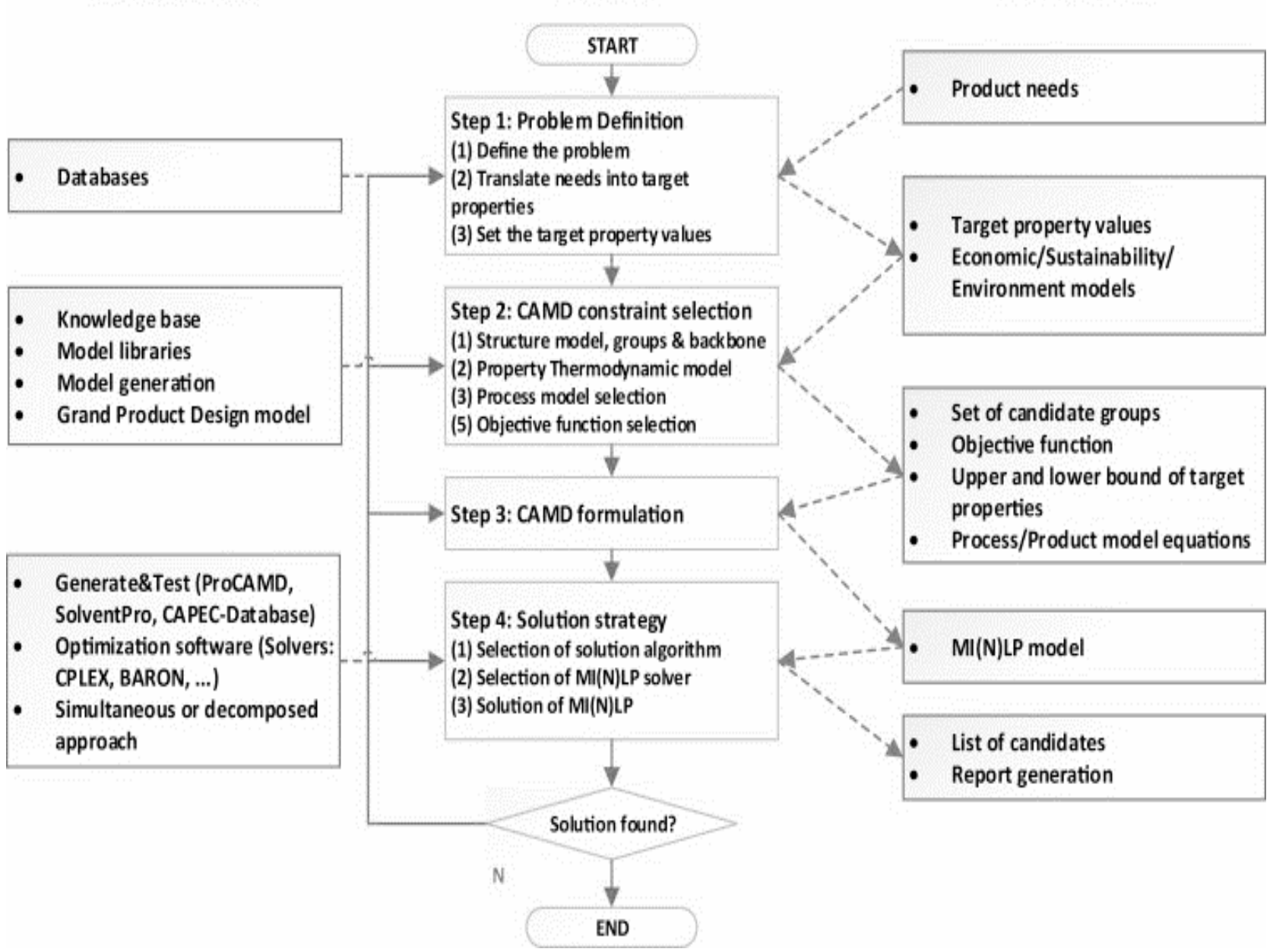

Figure 1 Computer-aided framework for chemical product design.

\subsubsection{Step 2 Computer-Aided Molecular Design Constraint Selection.}

In this step, the CAMD constraints are selected based on the problem definition. The constraints needed include

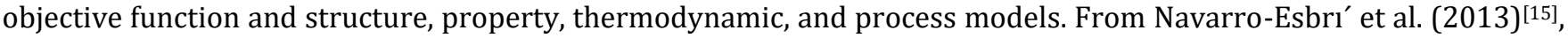
the ranges for condensation and evaporation temperatures are obtained:

evaporating temperature $\mathrm{T}_{\mathrm{e}}=272.9 \mathrm{~K}$

condensing temperature $\mathrm{T}_{\mathrm{cd}}=323.15 \mathrm{~K}$

mean process temperature $\mathrm{T}_{\mathrm{m}}=298 \mathrm{~K}$
$2-1$

Environmental, health and safety concerns are important issues for refrigerant design. The ozone depletion potential (ODP) and the tropospheric lifetime are related to the environmental impact of a compound. Non-flammability, good stability and low toxicity are also desirable. However, in this study, only the most fundamental thermodynamic criteria (heat of vaporization, liquid specific heat and vapor pressure) are considered in the optimization formulation. The environmental characteristics as well as the stability of the molecules designed are considered after the optimization problem has generated some candidate molecules.

The CAMD problem is formulated based on the collected properties of R134a in table 2, the design specifications for this problem are given in table 3. All the explicit target properties for this problem are in the CAMD methodology considered as secondary pure-component properties. In addition, we have included some implicit target properties, for example, the refrigerant should not polymerize over a very long period of time; it should not be explosive nor corrosive. Thus, with the help of our knowledge base on properties, we are able to preselect the groups are given in Table 4 see figure 2 . The new refrigerants must respect the following characteristics: 
Must not polymerize.

Be nonflammable.

Assure that if there are leaks, they are from the refrigeration system.

A small amount of refrigerant must allow a transfer of the heat.

A small amount of refrigerant must flash into the valve.

The following specifications are necessary to solve the problem through ProCAMD see figure 3:

To ensure that the refrigerant does not polymerize, compounds that have double or triple bonds will be avoided.

To ensure that the refrigerant is nonflammable, compounds involving both nitrogen and halides will be avoided.

To ensure that if there are leaks, they are from the refrigeration system, desirable refrigerant must have a vapor pressure between:

$$
\begin{aligned}
& \mathrm{Ps}^{\mathrm{Te}}>0.15 \mathrm{bar} \\
& \mathrm{P}_{\mathrm{s}}^{\mathrm{Tcd}}<15 \text { bar }
\end{aligned}
$$

To reduce the amount of refrigerant needed, the latent heat of vaporization must be as high as possible. The latent heat of vaporization of R134a is $24 \mathrm{KJ} / \mathrm{mol}$, so to assure that the new refrigerant is at least as good as the R134a and knowing that the latent heat of vaporization decreases with temperature, the refrigerant must check:

$$
\mathrm{H} v \text { at } \mathrm{T}_{\mathrm{e}}>22 \mathrm{KJ} / \mathrm{mol}
$$

To reduces the amount of refrigerant that flashes upon passage through the expansion valve, liquid specific heat must be as low as possible.

$$
\mathrm{CPl}_{\mathrm{Pl}} \text { at } \mathrm{T}_{\mathrm{m}}<180 \mathrm{~J} / \mathrm{mo} 1 \mathrm{~K}
$$

\subsubsection{Step 3 CAMD problem formulation}

In step 3, the constraint equations are collected to define the generic CAMD problem formulation as a mathematical programming problem. The general form of the MINLP is given by Eqs. (2-1)-(2-4). The CAMD problem includes integer variables, which come from the selection of molecular groups. The form of the CAMD problem (linear or nonlinear) depends on the choice of the property model and process model equations. The CAMD problem is now formulated in terms of the given the target properties, process constraints by retrieving the corresponding models from the model library of the framework as tables 3.

$\operatorname{Min} / \max \operatorname{obj}(\mathrm{X}, \mathrm{N})$

structural constraints: $\mathrm{g} 1(\mathrm{~N}, \mathrm{Y}) \leq 0$

property constraints: g2 $(\mathrm{N}) \leq 0$

process model and other constraints: $\mathrm{g} 3(\mathrm{X}, \mathrm{N})=0$

$X \in R n, N \in Z m, Y \in\{0,1\} q$

\section{2-8}


Table 2 R-134a Properties

\begin{tabular}{|l|l|}
\hline Property of R134a & Value \\
\hline Molecular weight & $102.03 \mathrm{~mol} / \mathrm{g}$ \\
\hline Normal boiling point & $247.15 \mathrm{~K}$ \\
\hline Critical temperature & $374.15 \mathrm{~K}$ \\
\hline Critical pressure & $40.59 \mathrm{bar}$ \\
\hline Acentric factor & 0.33 \\
\hline Enthalpy of Vaporization at Tb & $215.9 \mathrm{~kJ} / \mathrm{kg}$ \\
\hline ODP & 0 \\
\hline GWP & 1370 \\
\hline Thermal conductivity (liquid, Tb) & $0.103 \mathrm{~W} / \mathrm{m} \mathrm{K}$ \\
\hline Thermal conductivity (vapor, Tb) & $0.009 \mathrm{~W} / \mathrm{m} \mathrm{K}$ \\
\hline
\end{tabular}

Table 3 Target Product Properties

\begin{tabular}{|l|l|l|}
\hline Target Product Property & Lower Bound & Upper Bound \\
\hline Molecular mass & & $150 \mathrm{~g} / \mathrm{mol}$ \\
\hline Normal boiling point $^{\mathrm{a}}$ & $200 \mathrm{~K}$ & $400 \mathrm{~K}$ \\
\hline Critical temperature $^{\mathrm{a}}$ & $350 \mathrm{~K}$ & $450 \mathrm{~K}$ \\
\hline Critical pressure $^{\mathrm{a}}$ & $30 \mathrm{bar}$ & $50 \mathrm{bar}$ \\
\hline${\text { Enthalpy of Vaporization at } \mathrm{Tb}^{\mathrm{a}}}^{\mathrm{a}}$ & $200 \mathrm{~kJ} / \mathrm{kg}$ & \\
\hline Thermal conductivity (liquid, $\mathrm{Tb})^{\mathrm{a}}$ & $0.08 \mathrm{~W} / \mathrm{m} \mathrm{K}$ & 0 \\
\hline ODP & & 1400 \\
\hline GWP & & 10 \\
\hline Number of groups & 1 & 2 \\
\hline Number of functional groups & 0 & \\
\hline
\end{tabular}

Table 4 Preselected Groups for the Refrigerant Design Problem

\begin{tabular}{|l|l|}
\hline Base group & CH3 CH2 CH C \\
\hline Components containing chlorine & CH2CL CHCL CCL CH2CL2 CHCL2 CCL2 CCL3 \\
\hline Components containing fluorine & CF3 CF2 CF F \\
\hline $\begin{array}{l}\text { Components containing chlorine and } \\
\text { fluorine }\end{array}$ & CCL2F HCCL2F HCCLF CCLF2 HCCLF2 CCLF3 CCL2F2 \\
\hline Components containing ether & CH3O CH2O CH-O \\
\hline
\end{tabular}




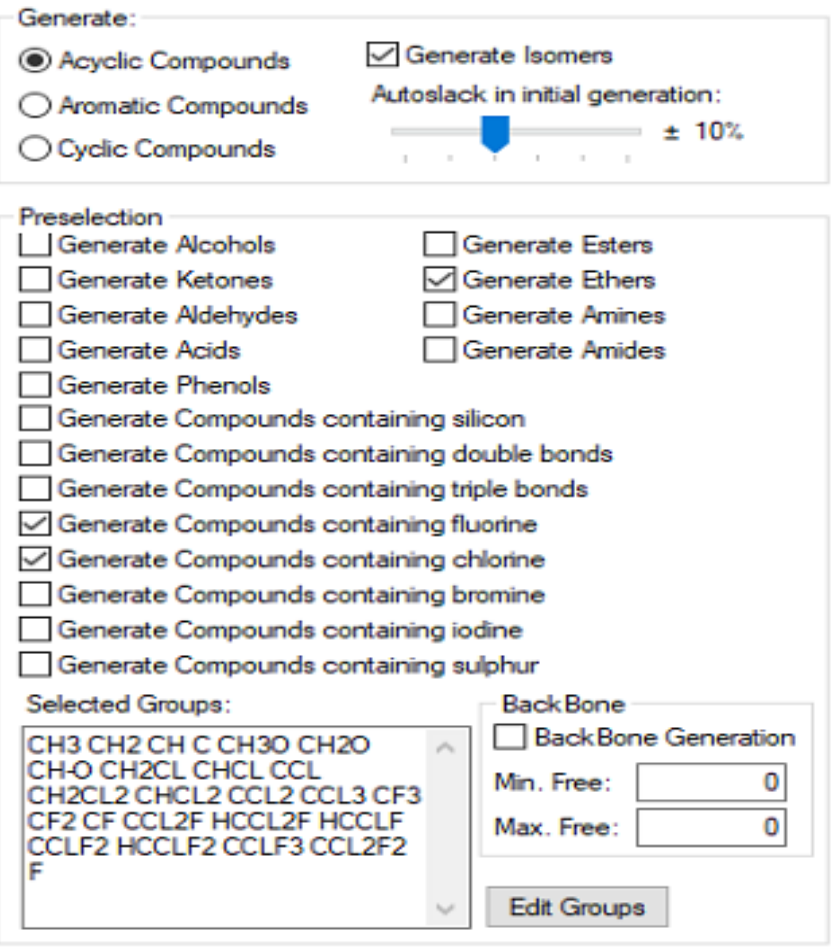

\begin{tabular}{|c|c|}
\hline & Delete \\
\hline & Define \\
\hline & Commom Solv. \\
\hline \multicolumn{2}{|l|}{ Extended Problem Control- } \\
\hline Minimum number of groups: & $2 \div$ \\
\hline Maximum number of groups: & $8 \div$ \\
\hline Minimum number of "functional" groups: & $0 \div$ \\
\hline Maximum number of "functional" groups: & $6 \div$ \\
\hline Minimum number of same "fiunctional" group: & $0 \div$ \\
\hline Maximum number of same "functional" group: & $6 \div$ \\
\hline \multicolumn{2}{|l|}{$\checkmark$ Perform Database search after generdion } \\
\hline$\square$ Calculate properties wth ProPred engine, a & intial screeening \\
\hline
\end{tabular}

Figure 2 CAMD preselect the groups

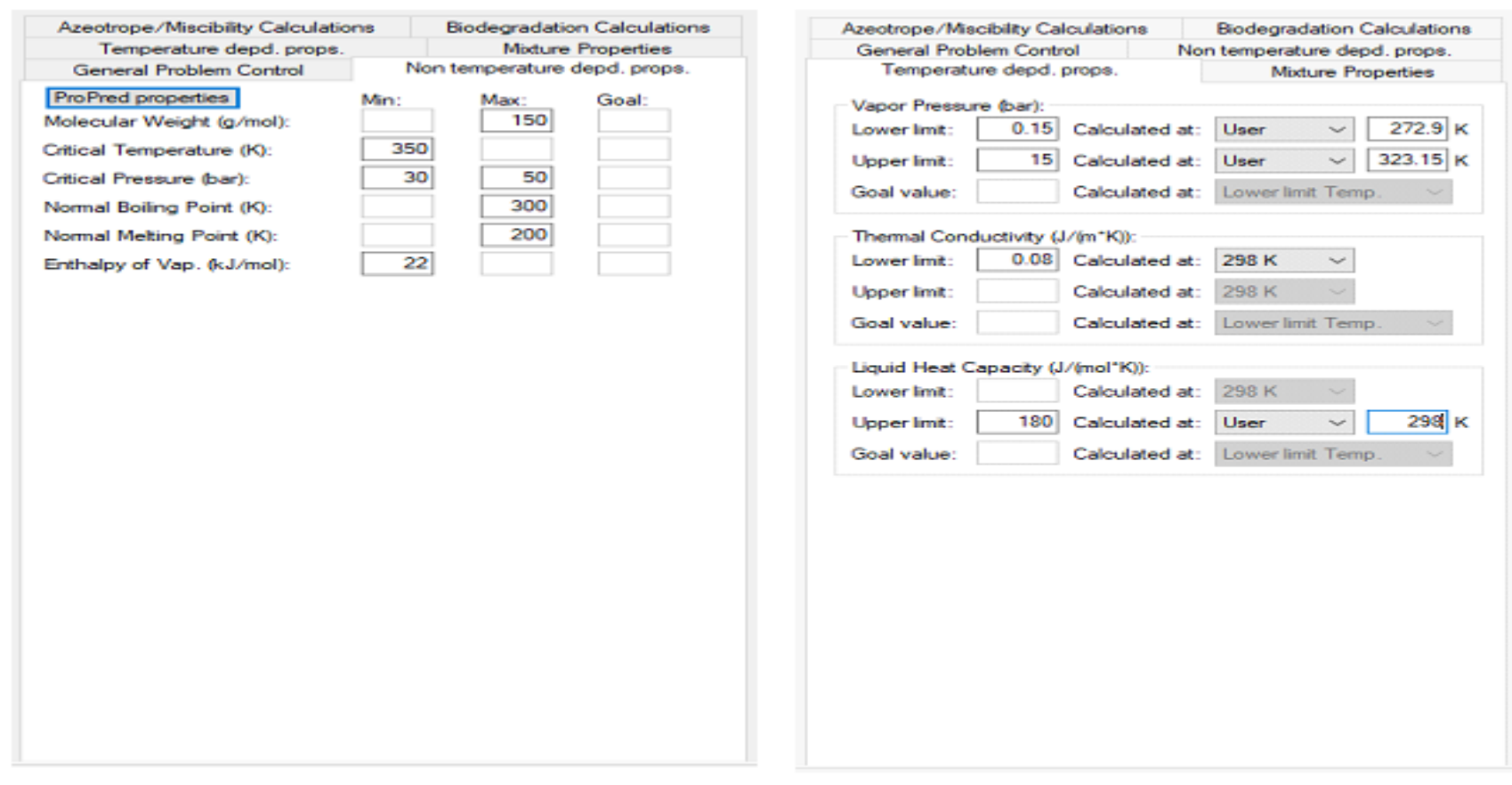

Figure 3 Target Product Properties 


\subsubsection{Step 4: Solution strategy}

Three options [database search, ProCAMD as well as MINLP (direct and decomposed)] are used to solve the problem. In this step, the solution strategy is chosen to solve the corresponding mathematical programming problem. Note that all three types of solution techniques are available through the framework, and in principle, any solution strategy can be applied to solve a problem. For example, a simple database search may be applied if experimentally measured data based solution is desired (ignoring, for example, the objective function), or, for a given set of functional groups, all structures satisfying the set of structural constraints are first generated and then tested for the target properties and then the process constraints. For the final feasible set of molecules, the objective function is calculated to identify the optimal, or all the constraints and the objective function may be solved together for the same set of functional groups through an appropriate numerical solver.

The solution strategies implemented in the framework and available in the VPPD-Lab product design simulator are shown in Fig. 4.

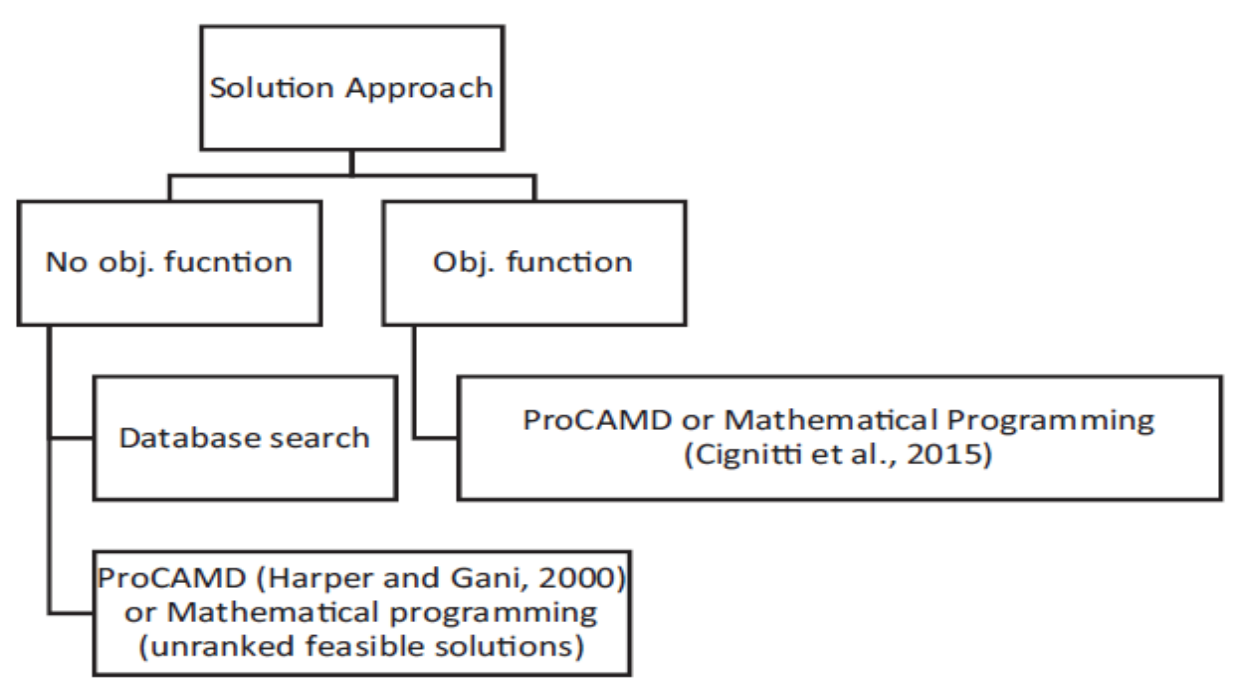

Figure 4 Solution strategies.

Database approach: This solution strategy is used when an objective function is either not defined or used and only pure component property targets are specified. In many cases, it is useful to solve a partial problem with only selected property constraints to get a good initial idea of the design problem search space see figure 5 .

ProCAMD approach: Here, the generate-and-test algorithm in [16] is employed to solve a wide range of single molecular product design problems. Any number of property constraints may be added as long as they are available in the property model library. Also, some implicit process constraints are possible to include. This approach generates (enumerates) all the feasible solutions within the defined search space. Finding the optimal in this case requires the calculation and ordering of the objective function values for the feasible molecules.

Mathematical programming approach: For this approach, two solutions options are available: direct solution and decomposed solution of MINLP problem. 


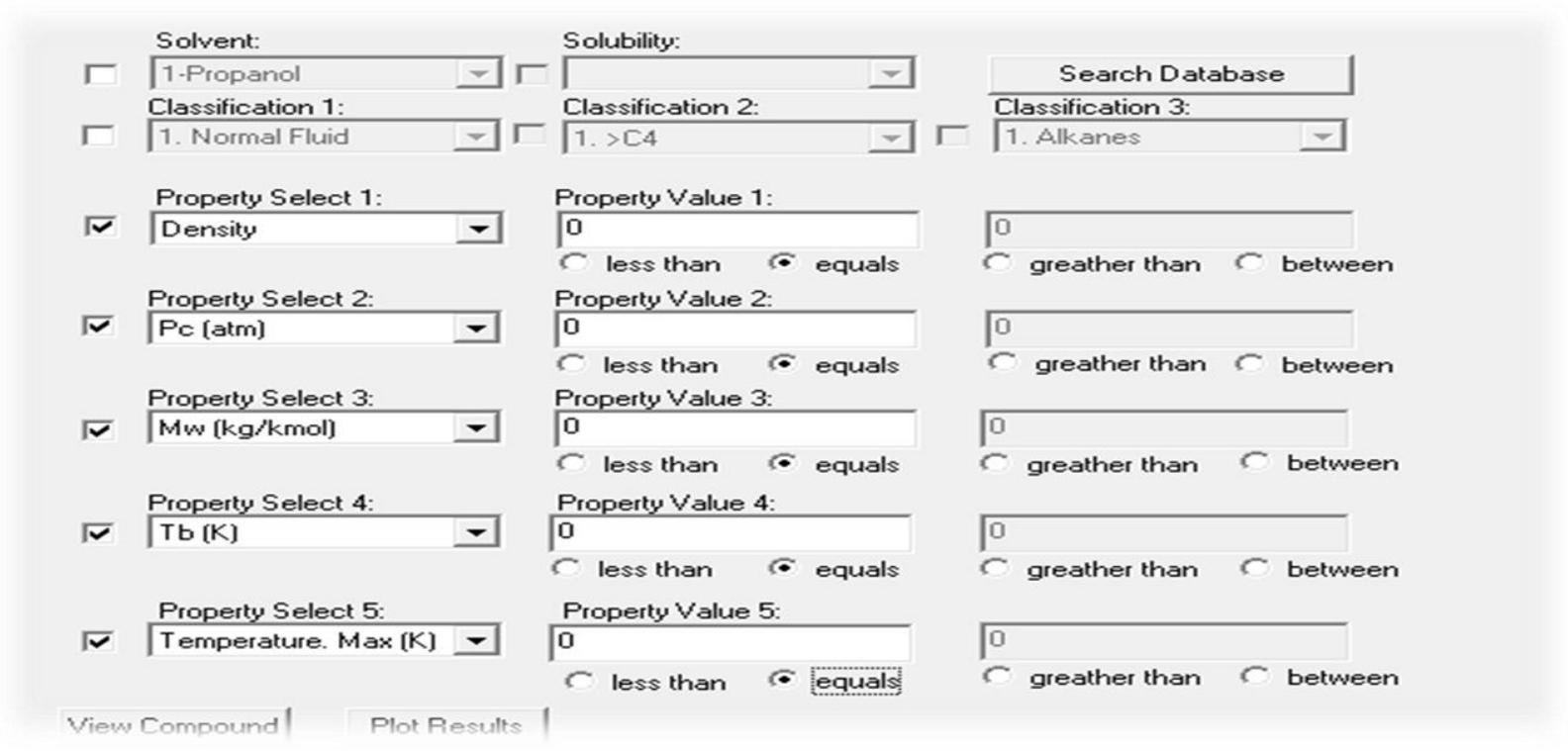

Figure 5 Database searcher screenshot

\subsection{Vapor Compression Refrigeration Cycle Applied in Aspen Hysys}

A description of the vapor compression refrigeration system is made as well as the propylene purification section of which the vapor compression refrigeration system is part of [17]. The work involves simulating the vapour compression refrigerating system in ASPEN Hysys V8.8 using molecules obtained from CAMD as the working fluids in different cases of the simulation.

\subsubsection{Process description of refrigeration unit}

The refrigeration unit uses R22 as a refrigerant. It operates on the principle of vapour compression refrigeration system with an evaporator, compressor, condenser and a throttling device (expansion valve) as its major components as shown in figure 6. The compressor is a screw type and has a designed adiabatic efficiency $80 \%$. The evaporator is a shell and tube heat exchanger with the refrigerant passing through the shell side and ethylene glycol passing through the tube side. The condenser is also a shell and tube type heat exchanger with the refrigerant going through the shell side and cooling water passing through the tube side. The expansion valve is a pressure-temperature letting device. The equipment reduces the pressure and temperature of the refrigerant thereby turning to liquid before entering the evaporator. The main function of the refrigeration unit in propylene purification process is to refrigerate glycol water from $4.6 \mathrm{C}^{\circ}$ to $-5 \mathrm{C}^{\circ}$. The refrigerated glycol water is used as a cooling medium in heat exchangers so as to recover propylene from being flared alongside in condensable. As the refrigerant enters the refrigeration circuit, it is allowed to flow through the expansion valve for pressure-temperature let down. The refrigerant flows into the evaporator and gains latent heat of vaporization from the glycol water and vaporizes, the compressor takes suction of the vaporized refrigerant at $-12.15 \mathrm{C}^{\circ}$ and compresses it, increasing both the pressure and the temperature of the refrigerant. The compressed refrigerant enters into the condenser where its temperature is reduced. At the outlet of the condenser is a high pressure liquid vapor mixture. The pressure and temperature is further reduced as the refrigerant undergoes expansion in the throttling device and the cycle is repeated again. 


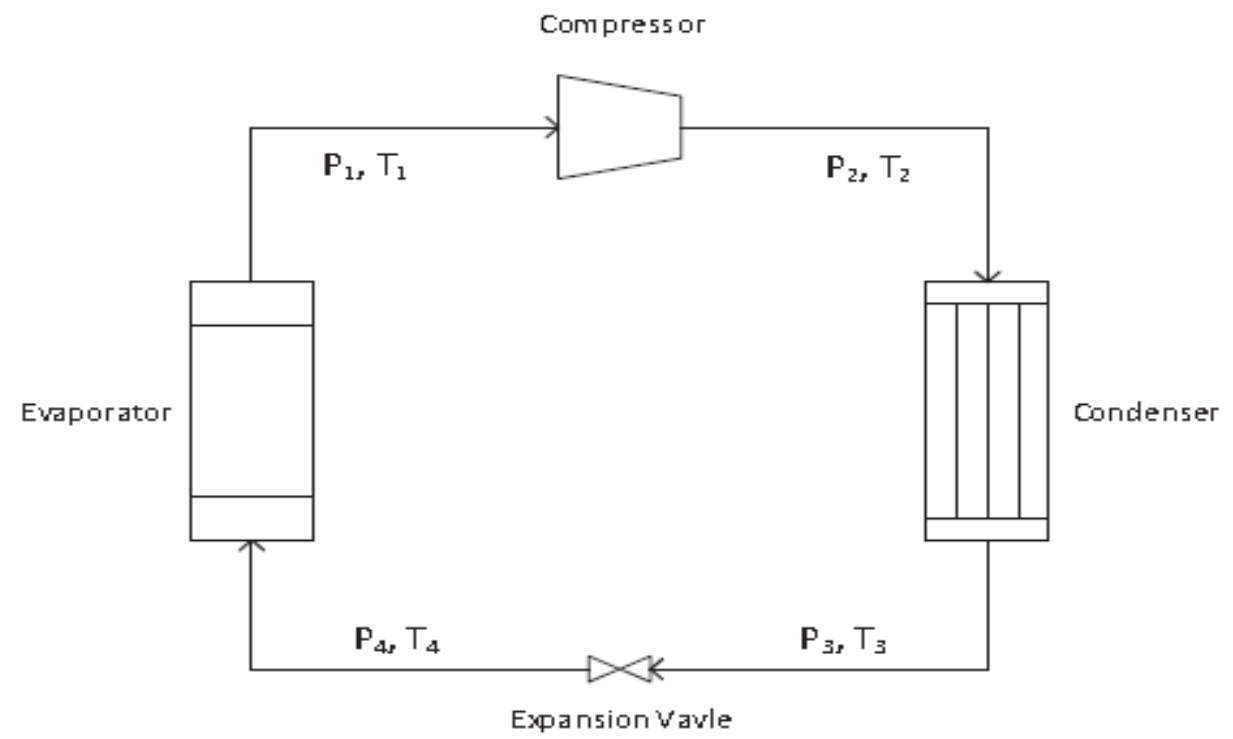

Figure 6 Schematic diagram of a vapor compression refrigeration system (VCRS)

\subsubsection{Simulation data source and model assumptions of VCRS}

In this study, the vapour compression refrigeration was simulated in AspenONE Engineering V8.0 software. The data used for simulating the refrigeration unit are operational data obtained from the propylene purification and refrigeration units. The software is a process design and optimization tool developed in 2012 and managed by Aspen Technology, Inc. It has many databases with more than 1800 components and 16000 binary interaction coefficients. The molecular obtained from CAMD use in Aspen and show in table 5.

Table 5 Simulation input data

\begin{tabular}{|l|l|l|l|l|}
\hline \multirow{2}{*}{ ID } & Molecule & \multicolumn{2}{l}{ Simulation input data } \\
\cline { 3 - 5 } & & Temperature & Flowrate & Composition fraction \\
\hline 1 & Ethyl trifluoromethyl ether & 261.15 & 7524 & 100 \\
\hline 2 & $1,1,1,2$-TetrafluoroEthane & 261.15 & 7524 & 100 \\
\hline 3 & 1 -Chloro-1,1,2-trifluoroethane & 261.15 & 7524 & 100 \\
\hline 4 & $1,1,1-$ Trifluoropropane & 261.15 & 7524 & 100 \\
\hline 5 & 1 -chloro-1,1-difluoropropane & 261.15 & 7524 & 100 \\
\hline 6 & $1,1,1,2$-tetrafluoropropane & 261.15 & 7524 & 100 \\
\hline 7 & $1,1,1-$ trifluoro-2-methylpropane & 261.15 & 7524 & 100 \\
\hline 8 & 1 -Chloro-1,1-difluoro-2-methylpropane & 261.15 & 7524 & 100 \\
\hline 9 & $1,1,1-$ TrifluoroButane & 261.15 & 7524 & 100 \\
\hline 10 & Cooling water & 280.15 & 46910 & 100 \\
\hline 11 & Ethylene glycol, Water solution & 277.8 & 45000 & $45,55 \%$ \\
\hline
\end{tabular}

The data used for the simulation of the refrigeration process are real time operational data gotten from available in published paper and operational manual of the polypropylene plan. The assumptions for modelling and simulating the refrigeration system is that: no pressure drop across the shell and tube of both the evaporator and condenser, the expansion process is assumed isenthalpic, no pressure losses in the piping and the compressor efficiency is $80 \%$.

The aim of the design performed for the vapor compression process is to identify the Coefficient of performance (COP) which represented by the following function: 
COP = Evaporator Duty/Compressor Power

The refrigerant absorbs heat in the evaporator ( $Q_{\text {ref }}$ ) also known as refrigerating effect and is expressed as:

$\mathrm{Q}_{\mathrm{ref}}=\mathrm{h}_{2}-\mathrm{h}_{1}$

$2-12$

The isentropic work input to the compressor is expressed as:

$\mathrm{W}_{\text {in }}=\mathrm{h}_{3}-\mathrm{h}_{4}$

Where $h_{1}, h_{2}, h_{3}$ and $h_{4}$ are enthalpies at point $1,2,3$ and 4 respectively.

The coefficient of performance is the refrigeration effect or evaporator duty (Qref) per unit compressor work or compressor duty (W) in the same thermal units.

Qref = refrigerating effect or evaporator duty.

$\mathrm{W}=$ Compressor work or compressor duty.

Coefficient of performance (C.O.P) is given by:

$\mathrm{COP}=\mathrm{Q}_{\mathrm{ref}} / \mathrm{W}$

The COP of a refrigeration system is a reflection of the system's energy performance is one of the key indicators for selecting a refrigerant as a possible replacement of R-22.

\section{Results and discussion}

\subsection{CAMD Design Results}

\subsubsection{Molecules Result}

Table 6 Molecular that exist in ProCamd.

\begin{tabular}{|l|l|}
\hline Formula & Name \\
\hline C3H5F30 & Ethyl trifluoromethyl ether \\
\hline C2H2F4 & 1,1,1,2-TetrafluoroEthane \\
\hline C2H2ClF3 & 1-Chloro-1,1,2-trifluoroethane \\
\hline C3H5F3 & 1,1,1-Trifluoropropane \\
\hline C3H5ClF2 & 1-chloro-1,1-difluoropropane \\
\hline C3H4F4 & 1,1,1,2-tetrafluoropropane \\
\hline C4H7F3 & $1,1,1$-trifluoro-2-methylpropane \\
\hline C4H7ClF2 & 1-Chloro-1,1-difluoro-2-methylpropane \\
\hline C4H7F3 & $1,1,1$-TrifluoroButane \\
\hline
\end{tabular}

1,1,1,2-TetrafluoroEthane which is the base of study and intended to replace, known refrigerant called R134a, have the highest enthalpy of fusion and the lowest viscosity. (1-Chloro-1,1,2-trifluoroethane) which is known refrigerant called $\mathrm{R} 133 \mathrm{~b}$, have the highest melting point temperature, critical temperature, enthalpy of vaporization at Tb and Thermal conductivity also have the lowest liquid heat capacity. (1,1,1-Trifluoropropane) which is known refrigerant called $\mathrm{R} 263 \mathrm{fb}$, have the lowest boiling point temperature, critical temperature, enthalpy of vaporization at $\mathrm{T}_{298}$ and Thermal conductivity. (1-chloro-1,1-difluoropropane) which is known refrigerant called R262, have the highest viscosity. (1,1,1,2-tetrafluoropropane) which is known refrigerant called R254eb, have the highest critical pressure and the lowest melting point temperature and enthalpy of vaporization at $\mathrm{T}_{\mathrm{b}}$. (1-Chloro-1,1-difluoro-2-methylpropane) which 
probably has not been reported before as a refrigerant, have the highest boiling point temperature and enthalpy of vaporization at $\mathrm{T}_{298}$, also have the lowest enthalpy of fusion. (1,1,1-TrifluoroButane) also which has not been reported before as a refrigerant, have the lowest critical Pressure.

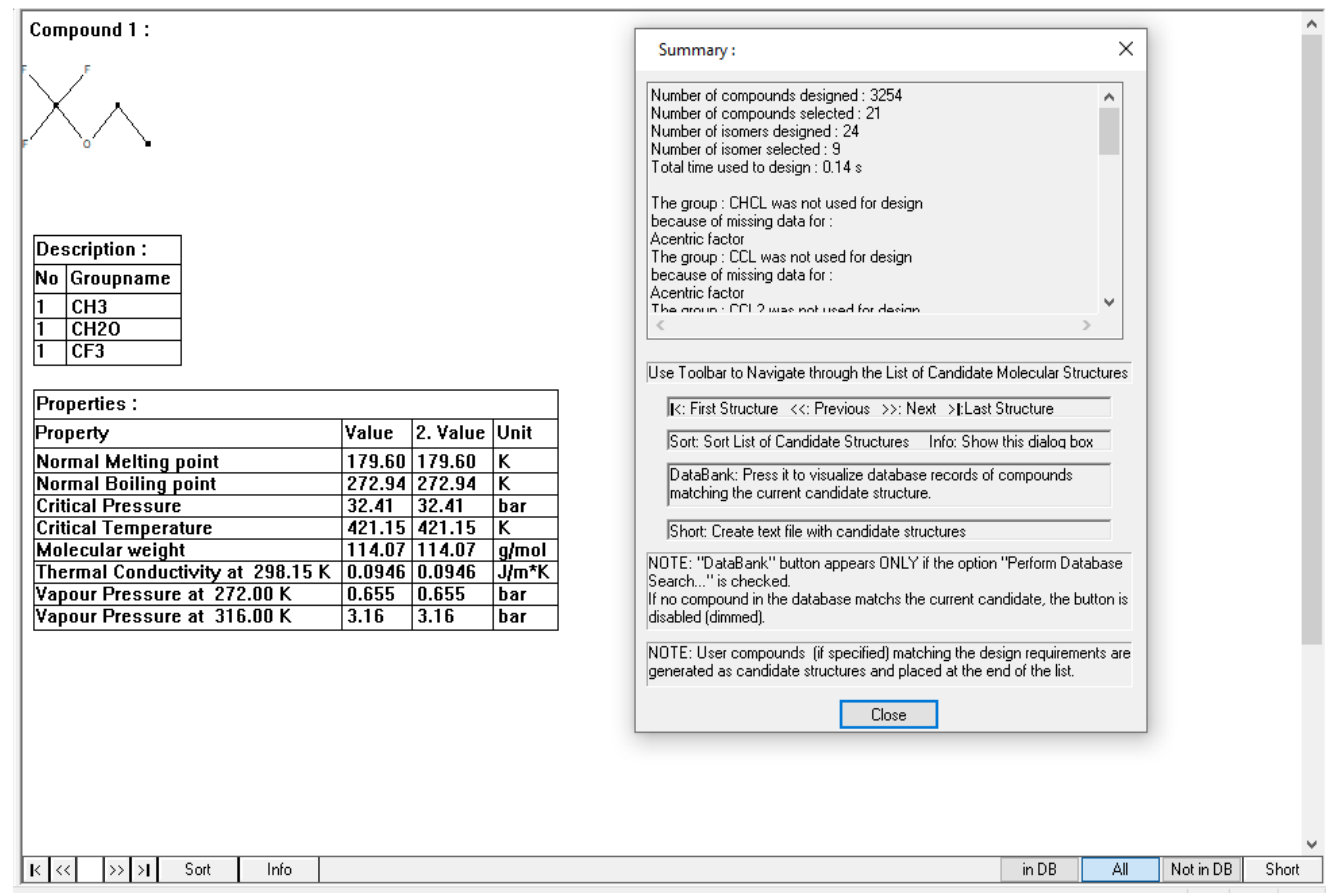

Figure 7 CAMD summary result.

\subsubsection{Safety and environmental performance of molecules}

Molecules that present favorable characteristics in all the performance indicators reported in Tables 6 can be selected and introduced to optimization of the VCR process.

Table 7 The environmental properties.

\begin{tabular}{|l|l|l|l|l|l|l|l|}
\hline ID & Name & $\mathbf{C}$ & $\mathbf{F}$ & Hv at Tb & $\begin{array}{l}\text { Cpl Jmol-1 K- } \\
\mathbf{1}\end{array}$ & ODP & GWP \\
\hline 1 & Ethyl trifluoromethyl ether & 1.68 & 0.483 & 23.40 & 168.637 & 0 & 15 \\
\hline 2 & $1,1,1,2-$-TetrafluoroEthane & 1.97 & NF & 25.16 & 138.389 & 0 & 1600 \\
\hline 3 & 1-Chloro-1,1,2-trifluoroethane & 0.96 & 0.396 & 31.20 & 137.996 & 0.06 & 870.9 \\
\hline 4 & 1,1,1-Trifluoropropane & 1.54 & 0.249 & 20.46 & 147.925 & 0 & 28.18 \\
\hline 5 & 1-chloro-1,1-difluoropropane & 1.02 & 0.344 & 26.68 & 151.354 & 0.02 & 25.12 \\
\hline 6 & 1,1,1,2-tetrafluoropropane & 1.82 & 0.566 & 17.32 & 158.674 & 0 & 67.61 \\
\hline 7 & $\begin{array}{l}1,1,1-\text {-trifluoro-2- } \\
\text { methylpropane }\end{array}$ & 3.17 & 0.309 & 22.94 & 170.926 & 0 & 738 \\
\hline 8 & $\begin{array}{l}1 \text {-Chloro-1,1-difluoro-2- } \\
\text { methylpropane }\end{array}$ & 1.47 & 0.759 & 27.98 & 175.891 & 0.3 & 49 \\
\hline 9 & 1,1,1-TrifluoroButane & \multicolumn{7}{|c|}{0.92} & 0.430 & 22.97 & 174.138 & 0 & 2.39 \\
\hline
\end{tabular}

\subsubsection{Criteria for performance evaluation of working fluids.}

An initial assessment of the performance indicators corresponding to the molecules reported in tables 7 leads to the following observations: 
The majority of the designed molecules indicate enthalpy of vaporization at $\mathrm{Tb}$ is higher than $22 \mathrm{KJ} / \mathrm{mol}$. As a result, the selection of molecules with considerably lower enthalpy of vaporization at $\mathrm{Tb}$ should also be avoided.

The majority of the designed molecules indicate liquid specific heat is lower than $180 \mathrm{Jmol}^{-1} \mathrm{~K}^{-1}$. As a result, the selection of molecules with considerably higher liquid specific heat should also be avoided.

Molecules with flammability greater than 0.6 will not be considered for further utilization as they are marked (strongly flammable) and should generally be avoided. On the other hand, molecules with flammability close to but less than 0.6 , have been considered for utilization in VCR cycle.

The desired toxicity levels of the selected molecules should be in the range of 2 or less, given that molecules utilized as VCR working fluids.

The coefficient of performance of the selected molecules should be as high as possible, especially coinciding with appropriate levels in all other employed performance measures.

With regards to the environmental performance of the designed molecules, Fluorinated ethers (Ethyl trifluoromethyl ether) present zero ODP and lower GWP values than hydrofluorocarbons, hence their favorable flammability and toxicological properties have made them a useful alternative. 1,1,1,2-TetrafluoroEthane is the base of study has high GWP, hence must be replaced. 1-Chloro-1,1,2-trifluoroethane was selected because the good properties. 1,1,1Trifluoropropane and 1,1,1,2-tetrafluoropropane were rejected, although it has zero ODP and low GWP because the enthalpy of vaporization is lower than $22 \mathrm{KJ} / \mathrm{mol}$. 1-chloro-1,1-difluoropropane and 1,1,1-TrifluoroButane represent zero ODP and GWP, hence these molecules has been selected. 1,1,1-trifluoro-2-methylpropane unaccepted because this molecular appears high toxicity, more than 2. 1-Chloro-1,1-difluoro-2-methylpropane also unconscionable because it considered strong flammable.

\subsection{Aspen Hysys Result}

\subsubsection{Model validation}

Model validation in table 8 was done by comparing coefficient of performance obtained from published paper in [17], with data gotten from the simulation.

Table 8 clarify model validation.

\begin{tabular}{|l|l|l|}
\hline $\begin{array}{l}\text { COP obtained from published paper } \\
\text { [simulation] }\end{array}$ & COP obtained from simulation & Error \% \\
\hline 3.634 & 3.428 & $94.33 \%$ \\
\hline
\end{tabular}

From table 8 above, the pressure differences between the plant and the simulation stems from the fact that the simulation assumes no pressure drop in the evaporator and condenser (for ease of simulation) whereas in reality, even though this equipment are not pressure let-down equipment, there is usually some pressure drop across them. The differences in temperature, pressure and even coefficient of performance for the plant and simulated condition may also be due to advances in software package used for the simulation. The simulation also assumes no pressure drop in the piping connecting this equipment but, there is usually pressure drop in any given length of pipe having a flowing fluid.

\subsubsection{Coefficient of Performance Result}

The results extracted from AspenHysys simulation as figure 8, for all the molecules selected from table 7 is displayed in Table 9 below. 
Table 9 Model validation, COP and model sensitivity analysis results

\begin{tabular}{|c|c|c|c|c|}
\hline ID & Molecules & $\begin{array}{l}\text { Evaporator } \\
\text { Duty }(\mathrm{kJ} / \mathrm{h})\end{array}$ & $\begin{array}{l}\text { Compressor } \\
\text { work }(k J / h)\end{array}$ & $\begin{array}{l}\text { Coefficient of } \\
\text { performance (COP) }\end{array}$ \\
\hline 1 & Ethyl trifluoromethyl ether & 1224185 & 270572 & 4.5 \\
\hline 2 & 1,1,1,2-TetrafluoroEthane (R134a) & 1224185 & 266160 & 4.6 \\
\hline 3 & 1-Chloro-1,1,2-trifluoroethane (R133b) & 1224185 & 175460 & 6.9 \\
\hline 4 & 1-chloro-1,1-difluoropropane (R262) & 1224185 & 329536 & 3.7 \\
\hline 5 & 1,1,1-TrifluoroButane & 1224185 & 442945 & 2.7 \\
\hline
\end{tabular}

From Table 9, all the investigated refrigerants under simulation produce refrigeration effect $1224185 \mathrm{~kJ} / \mathrm{h}$, COP of 1,1,1,2-TetrafluoroEthane base of study is 4.6, and COP of 1-Chloro-1,1,2-trifluoroethane represented the higher than that of other refrigerants under investigation.

\subsection{Identification of optimum VCR working fluid.}

From Table 9, it appears that the highest performing molecule in coefficient of performance terms is 1-Chloro-1,1,2trifluoroethane (R133b) which is known refrigerant. Although it has high COP, the last year production allowed in developed countries under Montreal protocol in 2029, for that reason it can't be considering alternative to R143a.

This is followed by Ethyl trifluoromethyl ether, represent fluorinated ether which has not been reported as a refrigerant before, can be alternative refrigerant.

1-chloro-1,1-difluoropropane which is known refrigerant called (R262). Although it has good result, the last year production allowed in developed countries under Montreal protocol in 2029, for that reason it can't be considering alternative to R143a.

1,1,1-TrifluoroButane which is new refrigerant shows a good environmental and toxicity data also acceptable coefficient of performance, therefor it can be consider alternative to R134a.

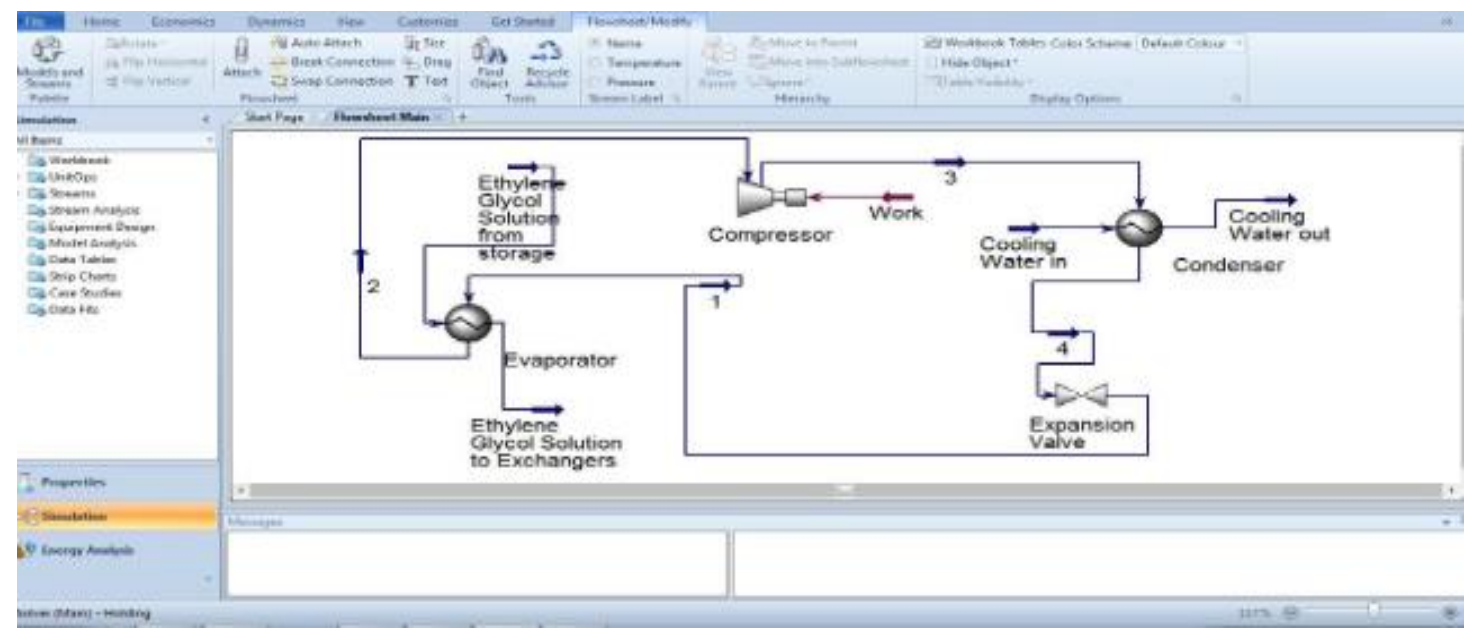

Figure 8 Screen Shot of VCRS Model with R-22 as Refrigerant

\section{Conclusion}

Accelerated technical development and economic growth throughout the world during the last century have produced severed environmental problems, forcing us to acknowledge that though these technological advances may contribute to human comfort, they also can threaten the environment through ozone depletion and global warming. The halocarbon refrigerants used in the refrigeration and air-conditioning systems have become a subject of great concern for the last few decades. The problem is not with refrigerants inside the system, but with their release to the 
environment. Earth is the only planet in the solar system with an atmosphere that supports life; therefore, preserving the ozone layer and reducing the release of greenhouse gasses to the atmosphere are part of the many essential steps necessary for the protection of life on the planet for future generations. CFCs and HCFCs were found harmful to the earth's protective ozone layer. Therefore, their production has been prohibited by the Montreal Protocol and other international agreements. HFC refrigerants are currently the leading replacement for CFC and HCFC refrigerants in refrigeration and air-conditioning systems. Therefore, this research discusses possibility of alternative fluids have good impact and environment friendly.

The problems and causes of ozone depletion and global warming. It proposed methodology indicate that the use of arbitrarily compiled databases for VCR working fluid selection significantly limits the opportunities of identifying highly performing options. The systematic design and subsequent selection of molecules based on evaluation of numerous relevant criteria:

- Leads to identification conventional working fluids covering a very broad range of potential performance characteristics.

- Enables the selection of working fluid options based on insights that are likely to reveal useful performance trade-offs.

- Points rapidly towards design options worth investigating, while avoiding the unnecessary consideration of sub-optimal choices.

\section{Recommendations}

The next generation refrigerants should be developed based on zero ODP and low GWP. In the future further research, regulation changes, the design of new systems suitable for the use of newly developed and natural refrigerants and the optimization of the system can be expected. There cannot be any ideal refrigerant but it is very important that the refrigerants should do less harm to the environment. The return to natural refrigerants at a new, high technology level should not be forgotten. The following are the important future research needs with respect to environment friendly alternative refrigerants:

- The return to natural refrigerants at a new, high technology level should not be forgotten.

- Hydrocarbon refrigerants will be considered as a long-term alternative for halogenated refrigerants, which are flammable. Hence, the development of new refrigeration system with low refrigerant inventory is essential.

- Very limited pure alternatives are available. Therefore, the new refrigeration system designs should accommodate the non-linear property variation of environment friendly mixed refrigerants.

- Compatibility of the alternative refrigerant mixtures with lubricants and the construction materials is required to be studied further.

- Inert nature of hydrocarbons with hydroflurocarbon refrigerants needs further investigation.

- An environmental property of new refrigerant mixtures also needs further investigation.

\section{Compliance with ethical standards}

\section{Acknowledgments}

I would like to thank Mr. Adil A. for his dedicated support and guidance. He continuously provided encouragement and was always willing and enthusiastic to assist in any way he could throughout the research project. I would also like to thank my colleague Mr. Saif Eddine G. for providing advice regarding simulation. Finally, many thanks to all participants that took part in the study and enabled this research to be possible.

\section{Disclosure of conflict of interest}

The authors have declared that no conflict of interest.

\section{References}

[1] Molina, M. J., \& Rowland, F. S., "Stratospheric sink for chlorofluoromethanes: chlorine atom-catalyzed destruction ozone" Nature 1974; 249:810-812. 
[2] Dekant, W., "Toxicology of chlorofluorocarbon replacements" Environmental Health Perspectives 104 (Suppl. 1), 1996; 75-83.

[3] Hayman, G., \& Derwent, R. D., "Atmospheric chemical reactivity and ozone-forming potentials of potential CFC replacements" Environ. Sci. Technol., 1997; 31 (2):327-336.

[4] Berends, A. G., de Rooij, C. G., Shin-Ya, S., \& Thompson, R. S., "Biodegradation and ecotoxicity of HFCs and HCFCs" Arch. Environ. Contam. Toxicol., 1999; 36:146-151.

[5] J. U. Ahamed, R. Saidur and H. H. Masjuki, "A review on exergy analysis of vapor compression refrigeration system”, Renew. Sust. Energy Rev., 2011; Vol. 15:pp. 1593-1600.

[6] N. E. Carpenter, "Retrofitting HFC 134a into existing CFC12 systems", Int.J. Refrig., 1992; Vol. 15(6):pp. 332-9.

[7] P. K. Bansal, Dutto and B. Hivet, "Performance evaluation of environmentally refrigerants in heat pumps 2: An experimental study with HFC 134a", Int. J. Refrig. 1992; Vol. 15(6):pp. 349-56.

[8] T.C., "Hung, Waste heat recovery of organic rankine cycle using dry fluids, Energy Conservation and Management", 2001; 42 (5):539.

[9] B.F. Tchanche, G. Papadakis, G. Lambrinos, A. Frangoudakis, "Fluid selection for a low-temperature solar organic rankine cycle" Applied Thermal Engineering 2009; 29:2468.

[10] T. Yamamoto, T. Furuhata, N. Arai, K. Mori,“Design and testing of organic rankine cycle”, Energy 2001; 26 (3):239.

[11] W.B. Stine, M. Geyer, Power from the sun, 2001. \{http:// www.powerfromthesun.net\}.

[12] friendly alternatives to halogenated refrigerants a review" International Journal of Greenhouse Gas Control 3, 108.

[13] M.T. Martin, M.D. Young, Prediction of the acute toxicity (96-h LC50) of organic compounds to the fathead minnow (Pimephales promelas) using a group contribution method, Chemical Research in Toxicology 2001; $14: 1378$.

[14] S. Kondo, Y. Urano, K. Tokuhashi, A. Takahashi, K. Tanaka, Prediction of flammability of gases by using F-number analysis, Journal of Hazardous Materials, 2001; 82- 113.

[15] Navarro-Esbri', J., Mendoza-Miranda, J.M., Mota-Babiloni, A., Barraga'n-Cervera, A., BelmanFlores, J.M. Experimental analysis of R1234yf as a drop-in replacement for R134a in a vapor compression system. International Journal of Refrigeration 2013; 36:870-880.

[16] Harper, P.M., Gani, R., "A multi-step and multi-level approach for computer aided molecular design", Computers and Chemical Engineering 2000; 24:677-683.

[17] Rilwan Omogbolahan Anjorin, Sheyin Israel Amos \& Sunday Kanshio, "Evaluation of Alternative Refrigerant for A $255000 \mathrm{kcal} / \mathrm{hr}$ 130kw Vapoure Compression Refrigeration System for Propylene Purification Process" International Journal of Engineering Sciences \& Research Technology. 2018. 